\section{Workload-indexed blood pressure response is superior to peak systolic blood pressure in predicting all-cause mortality}

\author{
Kristofer Hedman 1,2,3, Nicholas Cauwenberghs ${ }^{2,4}$, \\ Jeffrey W Christle ${ }^{1,2}$, Tatiana Kuznetsova ${ }^{4}$, Francois Haddad ${ }^{1,2}$ \\ and Jonathan Myers ${ }^{1,5}$
}

European Journal of Preventive Cardiology $0(00) 1-10$

(C) The European Society of Cardiology 2019

Article reuse guidelines: sagepub.com/journals-permissions DOI: 10.1 177/2047487319877268 journals.sagepub.com/home/cpr

@SAGE

\begin{abstract}
Aims: The association between peak systolic blood pressure (SBP) during exercise testing and outcome remains controversial, possibly due to the confounding effect of external workload (metabolic equivalents of task (METs)) on peak SBP as well as on survival. Indexing the increase in SBP to the increase in workload (SBP/MET-slope) could provide a more clinically relevant measure of the SBP response to exercise. We aimed to characterize the SBP/MET-slope in a large cohort referred for clinical exercise testing and to determine its relation to all-cause mortality.

Methods and results: Survival status for male Veterans who underwent a maximal treadmill exercise test between the years 1987 and 2007 were retrieved in 2018. We defined a subgroup of non-smoking 10-year survivors with fewer risk factors as a lower-risk reference group. Survival analyses for all-cause mortality were performed using Kaplan-Meier curves and Cox proportional hazard ratios (HRs ( $95 \%$ confidence interval)) adjusted for baseline age, test year, cardiovascular risk factors, medications and comorbidities. A total of 7542 subjects were followed over 18.4 (interquartile range 16.3) years. In lower-risk subjects $(n=709)$, the median (95th percentile) of the SBP/MET-slope was 4.9 (I0.0) $\mathrm{mmHg} / \mathrm{MET}$. Lower peak SBP $(<210 \mathrm{mmHg})$ and higher SBP/MET-slope $(>10 \mathrm{mmHg} / \mathrm{MET})$ were both associated with $20 \%$ higher mortality (adjusted HRs 1.20 (I.08-1.32) and 1.20 (I.I0-I.3I), respectively). In subjects with high fitness, a SBP/MET-slope $>6.2 \mathrm{mmHg} / \mathrm{MET}$ was associated with a $27 \%$ higher risk of mortality (adjusted HR 1.27 (I.I2-I.45)). Conclusion: In contrast to peak SBP, having a higher SBP/MET-slope was associated with increased risk of mortality. This simple, novel metric can be considered in clinical exercise testing reports.
\end{abstract}

\title{
Keywords
}

Mortality, clinical exercise testing, external workload, epidemiology

Received 17 July 2019; accepted 29 August 2019

\section{Introduction}

Blood pressure (BP) is routinely assessed and reported as an integral part of clinical exercise testing. ${ }^{1}$ Current scientific statements and guidelines by the American Heart Association ${ }^{1}$ and the American College of Sports Medicine $(\mathrm{ACSM})^{2}$ define an exaggerated systolic BP (SBP) response to exercise as a peak SBP of $\geq 210 \mathrm{mmHg}$ in males and $\geq 190 \mathrm{mmHg}$ in females. These thresholds approximate the 90th percentile in young, healthy subjects, ${ }^{3-5}$ but are not consistently associated with outcome. In fact, systematic reviews and meta-analyses ${ }^{6-8}$ suggest a complex and somewhat ambiguous association between the SBP response to exercise and mortality. In some populations, a high

\footnotetext{
'Stanford Cardiovascular Institute, Department of Medicine, Stanford University, Stanford, CA, USA

${ }^{2}$ Division of Cardiovascular Medicine, Department of Medicine, Stanford University, Stanford, CA, USA

${ }^{3}$ Department of Clinical Physiology and Department of Medical and Health Sciences, Linköping University, Linköping, Sweden

${ }^{4}$ Research Unit Hypertension and Cardiovascular Epidemiology, KU Leuven Department of Cardiovascular Sciences, University of Leuven, Belgium

${ }^{5}$ Division of Cardiology, Veterans Affairs Palo Alto Health Care System, Palo Alto, CA, USA
}

\section{Corresponding author:}

Kristofer Hedman, Department of Clinical Physiology and Department of Medical and Health Sciences, Linköping University, S-58I 85 Linköping, Sweden.

Email: Kristofer.Hedman@liu.se 
peak SBP has been related to a lower-risk of future cardiovascular events and all-cause mortality. ${ }^{9-12}$

Using absolute thresholds for SBP response to exercise is problematic due to the linear relationship between SBP (via cardiac output) and external workload, ${ }^{13-15}$ as higher achieved workloads implies higher level of fitness, which, in turn, is strongly associated with survival. ${ }^{16,17}$ Reporting SBP in relation to workload could account for this relationship and, therefore, provide a more robust and physiologically relevant measure than absolute peak SBP. Guidelines on exercise testing provide an estimated normative increase in SBP of $\sim 10 \mathrm{mmHg}$ / metabolic equivalent of task (MET), ${ }^{1,2}$ although this suggested value of the SBP/MET-slope was recently challenged. ${ }^{18}$ To our knowledge, the SBP/MET-slope has never been evaluated in a large clinical population, and its prognostic value is unknown.

The purpose of this study was to characterize the SBP/MET-slope, as a measure of workload-indexed SBP response to exercise, in a large clinical population of males referred for exercise testing and to determine its association with all-cause mortality.

\section{Methods}

\section{Study design and sample}

The Veterans Exercise Testing Study is an ongoing, prospective evaluation of US Veterans referred for exercise testing, designed to address exercise test, clinical and lifestyle factors and their associations to health outcomes. The study was approved by the Institutional Review Board at Stanford University, and all subjects gave written informed consent prior to undergoing the baseline examination. Male Veterans who underwent a treadmill exercise test at the Veterans Affairs Health Care System in Palo Alto, California between 1987 and 2007 were considered for inclusion $(n=9079)$. The exclusion criteria are detailed in Figure 1(a).

Demographic, clinical and medication information was obtained from each subject's computerized medical records just prior to the exercise test. This included information on previous cardiovascular and pulmonary disease, hypertension, hypercholesterolemia, diabetes mellitus, smoking status (current and past) and use of cardiac medications and family history of coronary disease. For details on the definitions used, see the online supplementary material (Methods 1.1. and Table S1).

We ascertained vital status of the participants as of July 20, 2018. We verified dates of death using the VA Beneficiary Identification and Record Locator System File, which has been estimated to be $95 \%$ complete and accurate. ${ }^{19}$ Follow-up time was the time from the exercise test to death or to the date when a subject was last verified to be alive.

\section{Exercise assessments}

Each subject underwent a standardized treadmill exercise test, using an individualized ramp protocol. Standard criteria for termination were used, including signs of inducible cardiac ischemia or a sustained drop in SBP. ${ }^{1,2}$ A $\mathrm{SBP}>250 \mathrm{mmHg}$ or a diastolic BP $>115 \mathrm{mmHg}$ were relative indications for test termination. ${ }^{1,2}$ Peak exercise capacity in METs was estimated automatically using standard ACSM equations. ${ }^{2}$ Agepredicted peak METs were calculated using a population-specific equation, as follows: $18-(0.15 \times$ age $) .{ }^{20}$

BP was measured by auscultation, standing at rest before the exercise test (SBPrest) and just prior to test termination (SBPpeak). The $\triangle \mathrm{SBP}$ was calculated as (SBPpeak - SBPrest) and indexed by the increase in METs from rest ( $\triangle$ METs; (peak METs - 1)) to obtain the SBP/MET-slope. For details on the exercise test measurements and calculations, see the online supplementary material (Methods 1.2).

\section{Subgroups and categories}

Lower-risk group. To explore the effect of comorbidities and risk factors on the SBP/MET-slope and to obtain 50th, 90th and 95th percentiles, we defined a subgroup of patients with lower cardiovascular risk. This group included non-smoking subjects surviving at least 10 years following the exercise test, without a history of diabetes mellitus, hypertension or cardiovascular disease. A detailed description of the selection of lowerrisk subjects is presented in the online supplementary material (Methods 1.3).

SBPIMET-slope versus peak MET categories. To determine the effect of the SBP/MET-slope on mortality in subjects with higher versus lower fitness, we defined four groups based on the median value of peak METs and of the SBP/MET-slope of the main sample: 1) higher MET/lower slope; 2) higher MET/higher slope; 3) lower MET/lower slope; and 4) lower MET/higher slope.

Hypotensive BP response group. Subjects with a $\Delta \mathrm{SBP} \leq 0 \mathrm{mmHg}$ or an exercise test terminated due to a sustained drop in SBP were not included in the main sample, but analyzed separately in the outcome analysis.

\section{Statistical analysis}

SPSS software v25.0 (IBM Corp, Armonk, NY, USA) was used for database management and statistical analysis. Survival analyses were performed using $\mathrm{R}$ Studio v1.1.456 (R Studio Inc., Vienna, Austria) 


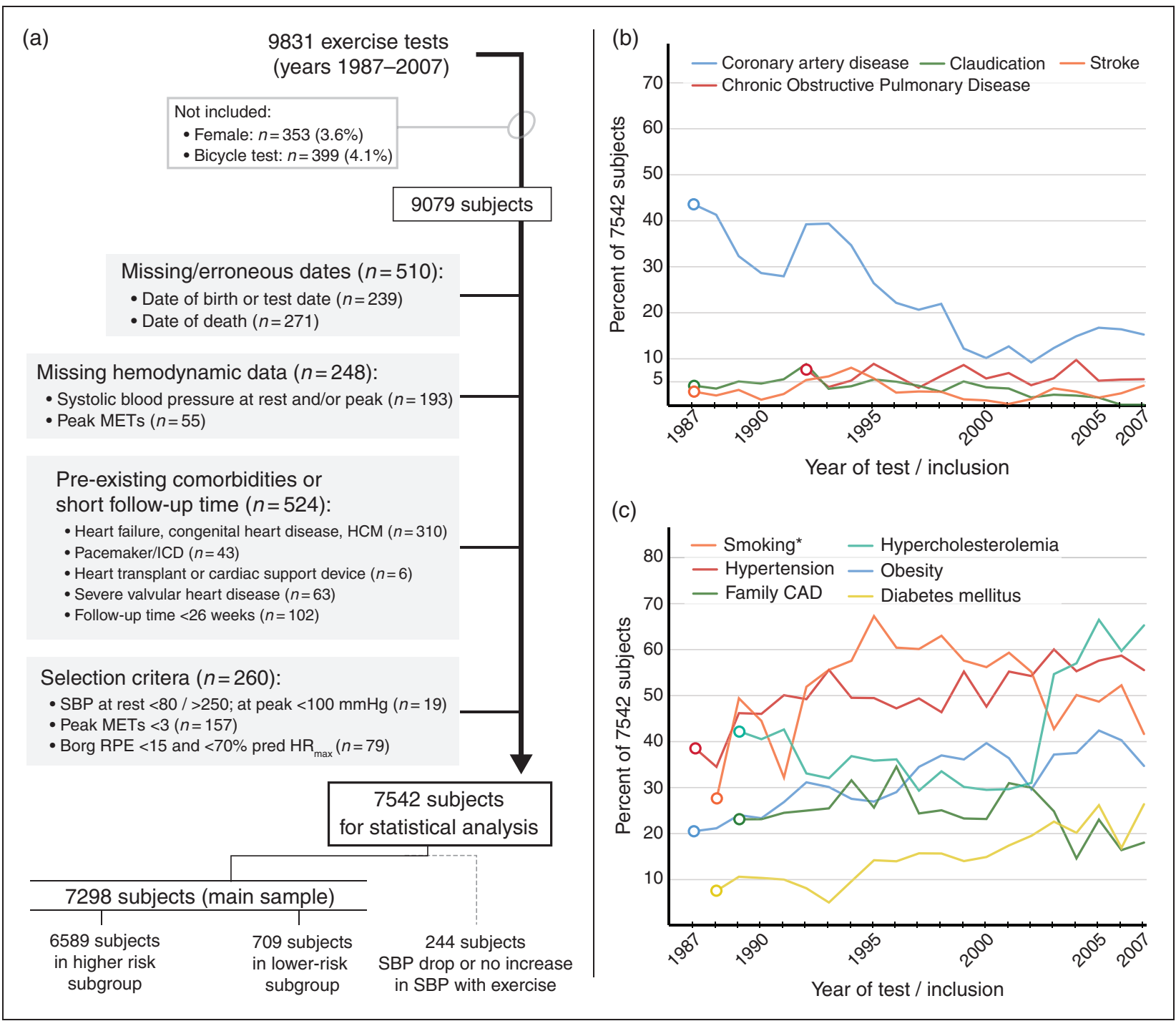

Figure I. Study flowchart (a) and comorbidities and cardiovascular risk factors over time. The 244 subjects with no increase or drop in SBP with exercise were only included in a separate survival analysis. In (b) and (c), the proportion (\%) of subjects with prevalent comorbidities (b) and different risk factors (c) per year of inclusion are visualized.

CAD: coronary artery disease; HCM: hypertrophic cardiomyopathy; ICD: implantable cardiac defibrillator; MET: metabolic equivalent of task; RPE: rating of perceived exertion; SBP: systolic blood pressure.

with the survival (v2.38) and survminer (v0.4.3) packages. Continuous variables were presented as mean (standard deviation) or median (interquartile range (IQR)) based on their distribution. We compared means, medians and proportions using Student's $t$-tests, Mann-Whitney U-tests and $\chi^{2}$ tests, respectively. Two-sided statistical significance was set at $<0.05$.

All outcome analyses were performed with 20-year all-cause mortality as the end-point. We applied the Kaplan-Meier method according to quartiles of the hemodynamic variables of interest, comparing survival over time using the log-rank (Mantel-Cox) test.
Unadjusted and adjusted Cox proportional hazard ratios (HRs) were calculated for quartiles of hemodynamic variables as well as for standardized continuous variables (Z-scores). Logistic regression was used to determine predicted probability of death within 20 years. The covariables included in the adjusted Cox models are detailed in the online supplementary material (Methods 1.4). Several sensitivity analyses were performed excluding subjects on cardiac medications and/ or different combinations of cardiovascular diseases. A landmark analysis set to year 1997 was performed to explore the stability over time in survival based on SBP/MET-slope. 
Table I. Clinical characteristics.

\begin{tabular}{|c|c|c|c|c|c|}
\hline & $\begin{array}{l}\text { Main sample } \\
(n=7298)\end{array}$ & $\begin{array}{l}\text { Lower-risk } \\
(n=709)\end{array}$ & $\begin{array}{l}\text { Higher risk } \\
(n=6589)\end{array}$ & $P^{*}$ & $\begin{array}{l}\text { Drop or no } \\
\text { increase in SBP with } \\
\text { exercise }(n=244)\end{array}$ \\
\hline Age (yrs) & $58.6 \pm 11.0$ & $53.4 \pm 12.2$ & $59.2 \pm 10.7$ & $<0.001$ & $64.4 \pm 10.2^{\dagger}$ \\
\hline Height (cm) & $176.3 \pm 7.9$ & $176.7 \pm 7.9$ & $176.3 \pm 7.9$ & 0.24 & $|75.| \pm 7.8^{\dagger}$ \\
\hline Body mass index $\left(\mathrm{kg} / \mathrm{m}^{2}\right)$ & $28.5 \pm 5.1$ & $27.6 \pm 4.5$ & $28.6 \pm 5.2$ & $<0.001$ & $27.4 \pm 4.6^{\dagger}$ \\
\hline Body surface area $\left(\mathrm{m}^{2}\right)$ & $2.05 \pm 0.20$ & $2.03 \pm 0.19$ & $2.05 \pm 0.20$ & 0.005 & $1.99 \pm 0.17^{\dagger}$ \\
\hline \multicolumn{6}{|l|}{ Risk factors ( $n, \%)$} \\
\hline Smoking & $3636(49.8)$ & 0 & $3636(55.2)$ & - & $107(43.9)$ \\
\hline Hypertension & 4291 (58.8) & 0 & $4291(65.1)$ & - & $167(68.4)^{\dagger}$ \\
\hline Diabetes mellitus & $1023(14.0)$ & 0 & $1023(15.5)$ & - & $44(18.0)$ \\
\hline Hypercholesterolemia & $2803(38.4)$ & $211(29.8)$ & $2592(39.3)$ & $<0.001$ & $82(33.6)$ \\
\hline Family history CAD & 1716 (23.5) & $156(22.0)$ & $1560(23.7)$ & 0.33 & $38(15.6)^{\dagger}$ \\
\hline \multicolumn{6}{|c|}{ Previous heart disease and comorbidities ( $n, \%)$} \\
\hline CAD & $1643(22.5)$ & 0 & $1643(24.9)$ & - & $116(47.5)^{\dagger}$ \\
\hline Stroke & $204(2.8)$ & 0 & $204(3.1)$ & - & $8(3.3)$ \\
\hline Claudication & $273(3.7)$ & 0 & $273(4.1)$ & - & $16(6.6)^{\dagger}$ \\
\hline Atrial fibrillation & $110(1.5)$ & 0 & $110(1.7)$ & - & $13(5.3)^{\dagger}$ \\
\hline COPD & $329(4.5)$ & 0 & $329(5.0)$ & - & $10(4.1)$ \\
\hline \multicolumn{6}{|l|}{ Current medication ( $n, \%)$} \\
\hline Anti-hypertensive & $3205(44.0)$ & 0 & $3205(48.6)$ & - & $140(57.4)^{\dagger}$ \\
\hline Beta-blocker & I494 (20.4) & 0 & I 488 (22.6) & - & $94(38.5)^{\dagger}$ \\
\hline Aspirin & 1717 (23.5) & 79 (II.0) & $1638(24.9)$ & $<0.001$ & $56(23.0)$ \\
\hline Statin & $659(9.0)$ & $38(5.4)$ & $620(9.4)$ & 0.001 & $22(9.0)$ \\
\hline
\end{tabular}

$* P$-value for difference between higher and lower cardiovascular risk groups.

${ }^{\dagger} p<0.05$ for comparison with main sample.

For details on the definitions used for each risk factor and comorbidity, readers are referred to the online supplementary methods.

SBP: systolic blood pressure; CAD: coronary artery disease; COPD: chronic obstructive pulmonary disease.

\section{Results}

\section{Study population characteristics}

A total of 7298 males (mean age $58.6 \pm 11.0$ years; 5th95th percentiles 40.6-76.3 years) were included in the main sample, of which 709 subjects were defined as having lower cardiovascular risk (Table 1). In addition, 244 subjects with a drop or no increase in SBP during exercise $(\Delta \mathrm{SBP} \leq 0 \mathrm{mmHg})$ were included for separate analysis. This group presented with a considerably higher prevalence of previous coronary artery disease (CAD) $(47.5 \%$ vs $22.5 \%, p<0.001)$ than other subjects. The risk factor profile and prevalence of comorbidities at the time of inclusion changed over the study enrollment period (Figure 1(b) and (c)). The unadjusted and adjusted relative risks of mortality associated with comorbidities, risk factors and use of medication are presented in the online supplementary material (Table S2).

\section{Hemodynamic data}

In total, $1152(15.8 \%)$ subjects had an exaggerated SBP response to exercise as defined by the guidelines (i.e. peak $\mathrm{SBP} \geq 210 \mathrm{mmHg}$ ). This group achieved higher absolute peak METs (as absolute and percentage of age-predicted), a higher rate of perceived exertion and a higher peak heart rate than those with peak SBP $<210 \mathrm{mmHg}$ (online supplementary material, Table S3). In addition, these subjects presented with higher systolic and diastolic BP at rest $(148 \pm 19$ vs $129 \pm 17$ and $88 \pm 11$ vs $80 \pm 11 \mathrm{mmHg}$, both $p<0.001)$ and lower prevalence of previous CAD than subjects with peak SBP $<210 \mathrm{mmHg}$.

Peak METs correlated weakly with SBP at rest $(r=-0.12, p<0.001)$ and with peak SBP $(r=0.16$, $p<0.001)$ and slightly stronger with $\triangle \mathrm{SBP}(r=0.29$, $p<0.001)$. The SBP/MET-slope showed a moderate negative correlation with peak METs $(r=-0.50, p<0.001)$, but this association was weaker in subjects within the middle two quartiles of fitness (i.e. 6.2-10.9 METs; online supplementary material, Table S4 and Figure S1).

The median (IQR) SBP/MET-slope in the lower-risk group was 4.9 (2.8) $\mathrm{mmHg} / \mathrm{MET}$, with a 95th and 97.5th percentile of 10.0 and $11.1 \mathrm{mmHg} / \mathrm{MET}$, respectively (Figure 2(a); online supplementary material, Table $\mathrm{S} 5)$. In the main sample, the median SBP/MET-slope 


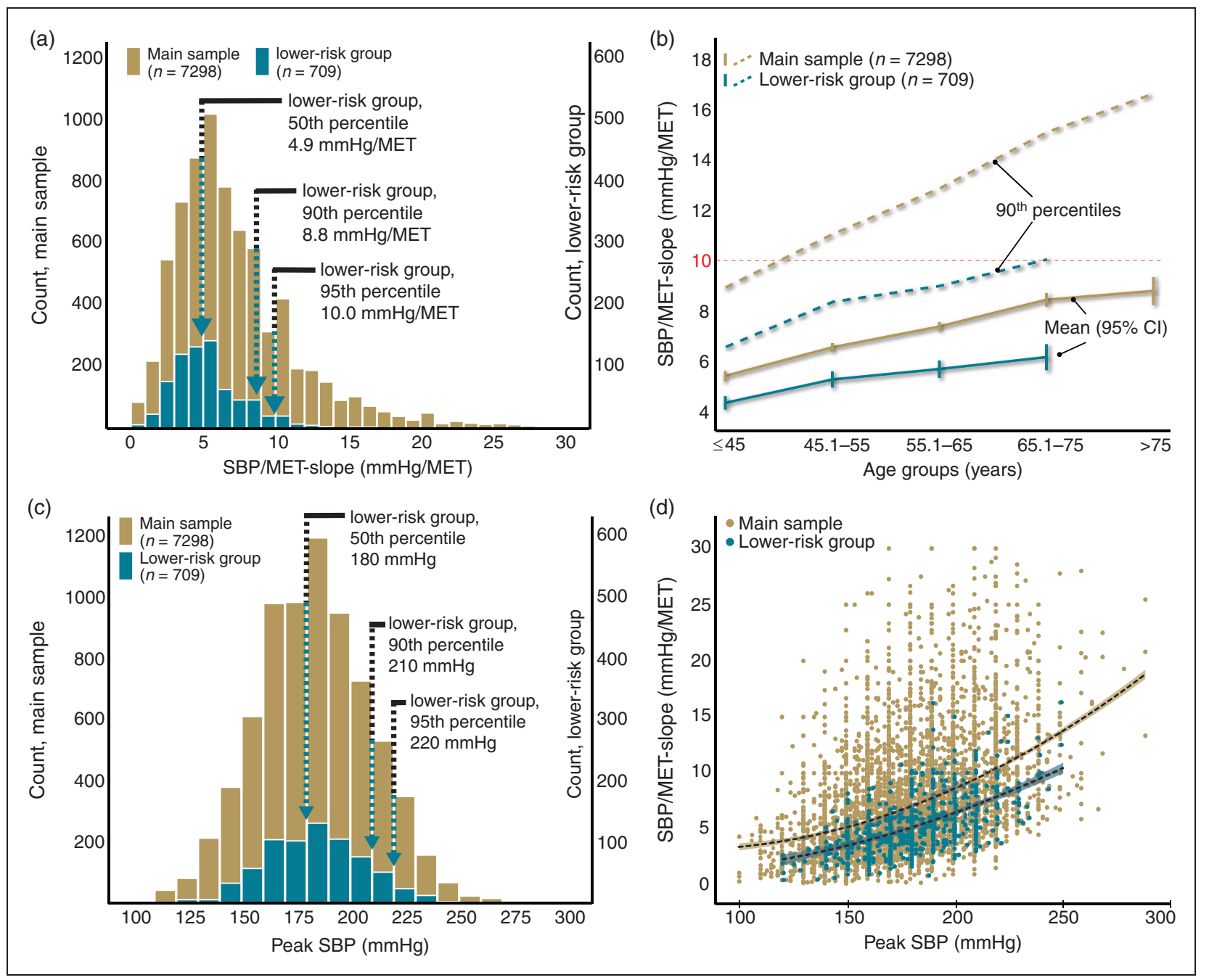

Figure 2. Peak SBP and SBP/MET-slope in the main sample (in gold) and in the subset of lower-risk subjects (in blue). The distribution of the SBP/MET-slope (calculated as $\triangle$ SBP/ $\triangle M E T$ ) and peak SBP in each group are illustrated in (a) and (c), where arrows denote the 50th, 90th and 95th percentiles in the lower-risk group. In (b), the mean value of the SBP/MET-slope (95\% Cls) as well as the 90th percentiles across age groups are presented. The red horizontal line represents the suggested average, normative value of $10 \mathrm{mmHg} /$ MET (as per American Heart Association guidelines). As only 18 subjects were $>\mathbf{7 5}$ years in the lower-risk group, no data are presented for this group. In (d), the SBP/MET-slope as a quadratic function of peak SBP is shown. In (a) and (d), 18 outliers ( $0.2 \%)$ in the main sample with a slope of $>30$ are not included.

was 6.2 (4.7) $\mathrm{mmHg} / \mathrm{MET} \quad$ (95th percentile: $16.0 \mathrm{mmHg} / \mathrm{MET})$. The SBP/MET-slope increased with age in both groups (Figure 2(b)).

\section{All-cause mortality and exercise hemodynamics}

In our cohort $(n=7542)$, the median (IQR) follow-up time was 18.4 years (16.3). During 138,546 person-years of observation, 3678 participants died (26.5 deaths per 1000 person-years).

SBP at rest and peak exercise and mortality. There was an increased risk of mortality for subjects in the two upper quartiles of SBP at rest (i.e. $>130 \mathrm{mmHg}$ ) in unadjusted analysis, but no difference between quartiles in fully adjusted analysis (online supplementary material, Figure S2). For peak SBP, subjects with lower peak SBP had greater risk of 20-year mortality during follow-up in both unadjusted and adjusted analyses (Figure 3). The HRs for each hemodynamic variable standardized as Z-score are presented in the online supplementary material (Table S6).

An excessive SBP response to exercise as defined by the guidelines (i.e. peak $\mathrm{SBP} \geq 210 \mathrm{mmHg}$ ) was not related to increased mortality in unadjusted analysis (HR 0.92, 95\% confidence interval (CI) 0.83-1.01, $p=0.07)$. When considering other risk factors, subjects without an excessive SBP response to exercise (i.e. peak 
SBP $<210 \mathrm{mmHg}$ ) had $20 \%$ higher relative risk of mortality (fully adjusted HR 1.20, 95\% CI 1.08-1.32, $p<0.001$ ) versus subjects with peak SBP $\geq 210 \mathrm{mmHg}$. After excluding subjects with atrial fibrillation or previous CAD, stroke, claudication in a sensitivity analysis, the fully adjusted $\mathrm{HR}$ associated with a peak SBP $<210 \mathrm{mmHg}$ was 1.17 (95\% CI 1.04-1.32, $p=0.010)$.

Subjects with a drop or no increase in SBP with exercise had a $88 \%$ (HR 1.88, 95\% CI 1.61-2.20, $p<0.001)$ higher unadjusted risk of all-cause mortality as compared with those with $\Delta \mathrm{SBP}>0 \mathrm{mmHg}$. After adjusting for comorbidities, risk factors and betablockers, there was a statistically non-significant $12 \%$ increase in risk associated with a drop or no increase in SBP (HR 1.12, 95\% CI 0.95-1.32, $p=0.17$ ).

SBPIMET-slope and mortality. Subjects with higher SBP/ MET-slope had greater cumulative risk of mortality during follow-up (Figure 3). In the highest quartile of the $\mathrm{SBP} / \mathrm{MET}$-slope (i.e. $>9 \mathrm{mmHg} / \mathrm{MET}$ ), 1028 subjects $(57 \%)$ died during 20 years of follow-up, compared with 703 subjects $(37 \%)$ in the lowest quartile (i.e. $\leq 4.3 \mathrm{mmHg} / \mathrm{MET}$ ). After adjusting for age, test year, body mass index and cardiovascular risk factors, medications and comorbidities, subjects with an SBP/ MET-slope in the highest quartile had a 23\% (HR 1.23, 95\% CI 1.12-1.36) higher risk of mortality than subjects in the lowest quartile. Excluding subjects with beta-blockers, anti-hypertensive medication, previous cardiovascular comorbidities or atrial fibrillation did not substantially alter these findings (sensitivity analyses; online supplementary material, Tables S7 and S8). Subjects with an SBP/METslope $>10 \mathrm{mmHg} / \mathrm{MET}$ (95th percentile in lower-risk group and by guidelines suggested normal, average increase) had a $69 \%$ (HR $1.69,95 \%$ CI 1.56-1.84) and $20 \%$ (HR 1.20, 95\% CI 1.10-1.31) higher risk of all-cause mortality versus those with $\leq 10 \mathrm{mmHg}$ /MET in the unadjusted and fully adjusted model, respectively. The relationship between SBP/MET-slope and mortality had a J-shape, with the lowest mortality for those with a slope around $4 \mathrm{mmHg} / \mathrm{MET}$ (online supplementary material, Figure S3). In a landmark analysis, overall survival increased by two years from the first to the second period of inclusion (landmark year 1997), but the prognostic pattern of SBP/MET-slope persisted (online supplementary material, Figure S4).

Fitness, SBPIMET-slope and mortality. Fitness was positively associated with survival in unadjusted and adjusted analyses (online supplementary material, Figure S2). When stratified by fitness, higher SBP/MET-slope (i.e. $>6.2 \mathrm{mmHg} / \mathrm{MET}$ ) was associated with worse unadjusted survival in subjects with higher fitness (>7.3 $\Delta$ MET, $p<0.001$ ), but not in unfit subjects
( $\leq 7.3 \Delta$ MET, $p=0.73$; online supplementary material, Figure S5). When adjusting for peak METs in continuous analysis of hemodynamic variables based on Z-scores, most HRs were statistically non-significant (online supplementary material, Table S6).

\section{Discussion}

This study demonstrated in a large cohort of male Veterans that higher workload-indexed SBP response to exercise rather than higher peak SBP was predictive of all-cause mortality. We also observed that in a subset of lower-risk subjects, a $10 \mathrm{mmHg}$ increase in SBP per MET corresponded to the upper 95th percentile rather than an average value as proposed by current exercise testing guidelines.

\section{Reappraisal of the normal SBP response to exercise}

During lower- or whole-body exercise, external workload is closely related to cardiac output and SBP, confirmed by early invasive studies. ${ }^{13,15,21}$ Accordingly, in male athletes reaching maximal effort, ${ }^{22}$ upper limits of normality in peak exercise SBP have been reported to be higher than in the general population. ${ }^{3,5}$ However, the normal workload-indexed response in SBP with exercise is not well defined. ${ }^{18}$ Although current guidelines on exercise testing suggest that SBP increases, on average, by $10 \mathrm{mmHg} / \mathrm{MET}$ during graded exercise, ${ }^{1,2}$ the underlying evidence is equivocal, and was recently questioned. ${ }^{18}$ In a subgroup of 709 subjects with lower cardiovascular risk and surviving at least 10 years from baseline, we observed $5 \mathrm{mmHg} / \mathrm{MET}$ and $10 \mathrm{mmHg} / \mathrm{MET}$ to represent the 50th and 95th percentile, respectively. Notably, we also observed a median $\mathrm{SBP} / \mathrm{MET}$-slope of $6.4 \mathrm{mmHg} / \mathrm{MET}$ in the remaining 6589 subjects with higher baseline risk. Our data suggest that the proposed normal increase in SBP per MET of $10 \mathrm{mmHg} / \mathrm{MET}^{1,2}$ represent an upper limit rather than an average expected increase.

\section{Peak SBP and mortality}

The definition and consequence of an exaggerated BP response with exercise remain controversial, ${ }^{4,18,23}$ and is more consistently reported as predictive of future development of hypertension ${ }^{7,24-27}$ than of cardiovascular risk and future mortality. ${ }^{6,11,28}$ Comparison across studies is challenging, considering the inconsistencies that exist in definitions and terminology for BP response to exercise. $^{1,29-31}$. In addition, SBP determined at a submaximal $^{24,28}$ or at peak external workload ${ }^{11,25,26}$ have been used to define thresholds. We found the adjusted relative risk of all-cause mortality to be $20 \%$ greater in subjects who did not reach a peak $\mathrm{SBP} \geq 210 \mathrm{mmHg}$ 


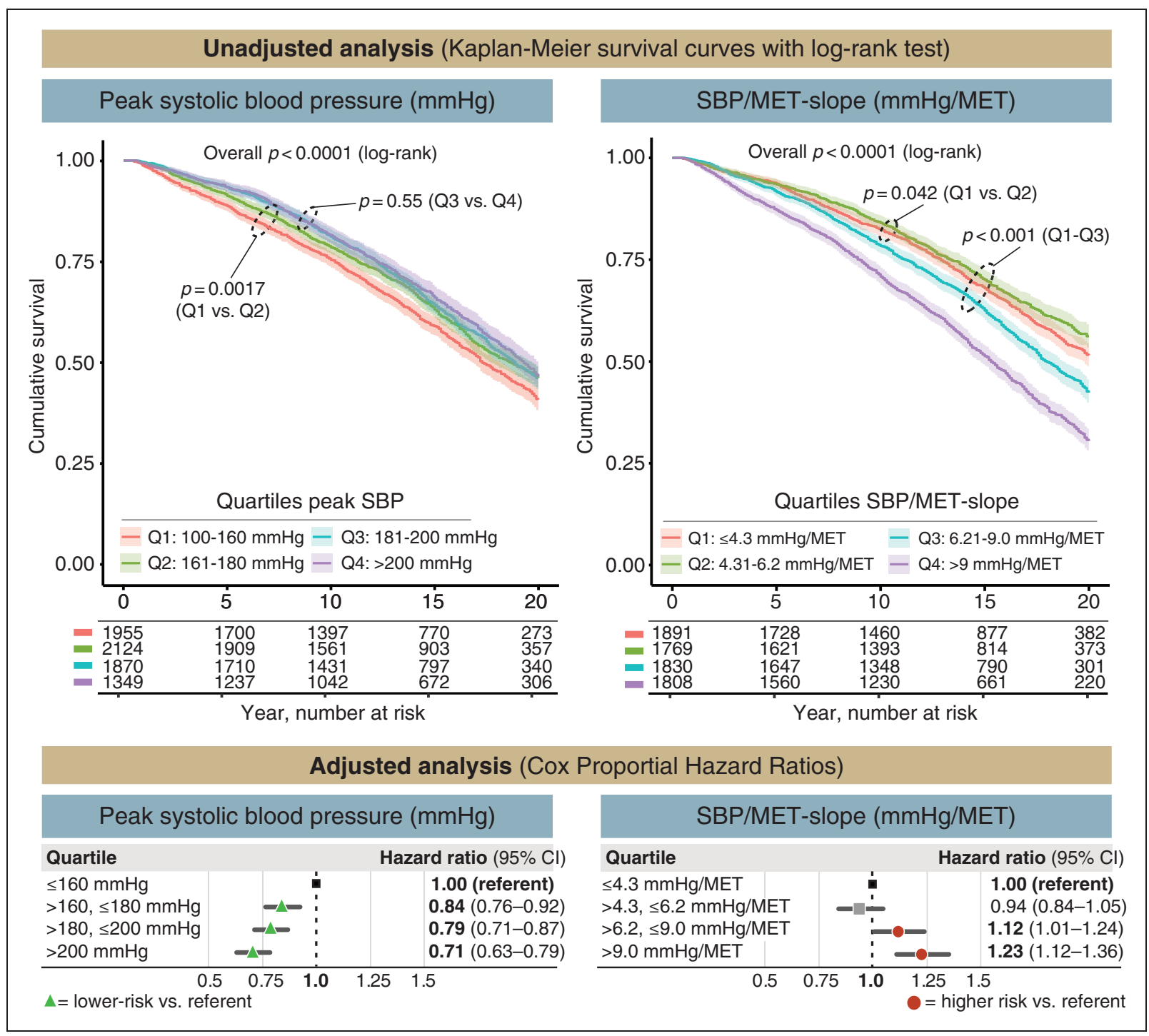

Figure 3. Cumulative survival and relative risk of death over 20 years per quartile of peak SBP and SBP/MET-slope. Kaplan-Meier curves (with 95\% Cls shaded) and Cox proportional HRs illustrating higher mortality in lower quartiles of peak SBP (left side) and in higher quartiles of the SBP/MET-slope (right side). HRs adjusted for test year, age, body mass index, cardiovascular risk factors, medications and comorbidities, as noted in methods.

MET, metabolic equivalent of task.

during exercise. This risk was still $16 \%$ higher after excluding subjects with known baseline cardiovascular disease. However, it is possible that a significant proportion of subjects had undiagnosed, subclinical cardiovascular disease, which could manifest itself as an inability to adequately increase SBP during exercise. Nevertheless, our data suggest that in a clinical referral sample, reaching the threshold defined as an exaggerated SBP response per current guidelines is not necessarily associated with increased risk of mortality in males.

One important factor to consider when reviewing previous literature on the SBP response to exercise and its association with outcomes is the baseline risk factor profile of the population being studied. In a study on 4907 males free from known heart disease and with a negative exercise electrocardiogram, Filipovský et al. ${ }^{32}$ found an increased relative risk of all-cause and cardiovascular mortality with higher $\triangle$ SBP during bicycle exercise testing. Similarly, in a highly selected sample of 300 normotensive, asymptomatic subjects undergoing clinical treadmill testing at the Mayo Clinic, the adjusted odds ratio for a major cardiovascular event within $7.7 \pm 2.9$ years of testing was $3.62(p=0.03)$ in subjects with a peak exercise SBP of $\geq 214 \mathrm{mmHg}(n=150)$, compared with those with a peak SBP of $170-192 \mathrm{mmHg}(n=150) .{ }^{30}$ In contrast, studies including subjects with verified or high risk of 
CAD have shown better ${ }^{9}$ or similar ${ }^{12}$ survival in subjects reaching a peak SBP $\geq 210 \mathrm{mmHg}$ than in subjects with lower peak SBP. Moreover, Hedberg et al. ${ }^{10}$ showed that in 372 community-dwelling 75 -year-old subjects, those with the highest $\triangle \mathrm{SBP}$ at the baseline exercise test $(\geq 55 \mathrm{mmHg})$ had the lowest risk of allcause and cardiovascular mortality during an average follow-up time of 10.6 years. In the current study, $23 \%$ of the sample had a history of CAD, while $2.8 \%$ and $3.7 \%$ had a previous diagnosis of stroke or claudication, respectively. Notably, the proportion of subjects with known CAD referred for exercise testing declined from $\sim 40 \%$ during the early years of study inclusion (1987-1988) to $\sim 10 \%$ around the year 2000 (Figure 1). However, after excluding subjects with previous cardiovascular disease, the relationships between low $\triangle \mathrm{SBP}$ or a high SBP/MET-slope and mortality persisted.

\section{The impact and rationale of considering external workload for risk prediction}

In contrast to the lower mortality associated with a higher peak SBP or $\triangle \mathrm{SBP}$ in our subjects, indexing the increase in SBP to external workload revealed an inverse relationship as a greater SBP/MET-slope was associated with higher mortality. Having an SBP/ MET-slope over $10 \mathrm{mmHg} / \mathrm{MET}$ was associated with $69 \%$ and $20 \%$ higher risk of 20 -year all-cause mortality in unadjusted and adjusted analyses, respectively. Given the strong association between fitness and survival, ${ }^{1,16,17}$ and the near-linear relationship between external workload and SBP, ${ }^{13-15}$ this is somewhat expected. In fact, studies measuring SBP at a defined submaximal workload have shown an association between submaximal SBP measures and mortality, ${ }^{28,33}$ as well as with incident myocardial infarction ${ }^{34}$ and stroke. $^{35}$ Using a meta-analytic approach, Schultz et al. ${ }^{6}$ showed that SBP at a submaximal intensity significantly increased the risk of experiencing a composite of cardiovascular event and/or death by $4 \%$ per $10 \mathrm{mmHg}$, while SBP at peak exercise was not associated with higher risk. Thus, relating exercise SBP to workload, either as SBP measured at a defined submaximal workload or as the SBP/MET-slope as in our study, seems superior in terms of prognostic capability. The SBP/MET-slope offers a method more applicable to ramp protocols, where precise measures at a specified submaximal workload may be difficult to accurately obtain.

The current study also underscores the strong relationship between fitness and survival, ${ }^{1,16,17}$ as peak METs was a strong determinant of survival, and adjusting for peak METs in the continuous analysis of Zscores rendered most HRs non-significant. However, the SBP/MET-slope added precision in terms of predicting survival in subjects with higher fitness. Specifically, in subjects achieving a peak METs value $\geq 8.2$ (the median in the main sample), having an SBP/ MET-slope $\geq 6.2$ was associated with $27 \%$ higher adjusted risk of all-cause mortality.

\section{Limitations}

First, our subjects were recruited as a clinically referred cohort and were all male; thus, these results are not necessarily representative of a general population or females. Second, measures of cardiac output and oxygen uptake were unavailable, and we relied on estimated external workload (METs) from standard, validated ACSM formulas. ${ }^{2}$ Third, submaximal measures of SBP were unavailable, and could have provided additional insight to the SBP response to exercise. Finally, we only had data on all-cause mortality, and our results call for studies exploring the association between SBP/ MET-slope and cardiovascular end-points.

\section{Conclusions}

Subjects exceeding the threshold defined as an exaggerated SBP response to exercise had better survival, while higher SBP/MET-slope was associated with worse survival. The SBP/MET-slope may add prognostic precision, at least among subjects with higher fitness, and could be considered for integration into clinical exercise testing reports.

\section{Author contribution}

FH and JM both contributed equally as senior authors in this work. KH, FH and JM contributed to the conception or design of the study. JM acquired the data and managed the database. KH, NC, TK, JWC, FH and JM contributed to the interpretation of data for the work. KH drafted the manuscript. All authors critically revised the manuscript. All gave final approval and agree to be accountable for all aspects of work ensuring integrity and accuracy.

\section{Declaration of conflicting interests}

The author(s) declared no potential conflicts of interest with respect to the research, authorship and/or publication of this article.

\section{Funding}

The author(s) disclosed receipt of the following financial support for the research, authorship, and/or publication of this article: this work was supported by general funding through the Stanford Cardiovascular Institute and post-doctoral research grants directed to $\mathrm{KH}$ from the Swedish Fulbright Commission; the Swedish Medical Society (SLS-682871); the Swedish Heart Foundation; and the County Council of Östergötland, Sweden (LIO-804111). 


\section{References}

1. Fletcher GF, Ades PA, Kligfield P, et al. Exercise standards for testing and training: a scientific statement from the American Heart Association. Circulation 2013; 128(8): 873-934.

2. ACSM's guidelines for exercise testing and prescription, 10th ed. Philadelphia, PA: Wolters Kluwer Health, 2018.

3. Daida H, Allison TG, Squires RW, et al. Peak exercise blood pressure stratified by age and gender in apparently healthy subjects. Mayo Clin Proc 1996; 71(5): 445-452.

4. Le VV, Mitiku T, Sungar G, et al. The blood pressure response to dynamic exercise testing: a systematic review. Prog Cardiovasc Dis 2008; 51(2): 135-160.

5. Sabbahi A, Arena R, Kaminsky LA, et al. Peak blood pressure responses during maximum cardiopulmonary exercise testing: reference standards from FRIEND (Fitness Registry and the Importance of Exercise: A National Database). Hypertension 2018; 71(2): 229-236.

6. Schultz MG, Otahal P, Cleland VJ, et al. Exerciseinduced hypertension, cardiovascular events, and mortality in patients undergoing exercise stress testing: a systematic review and meta-analysis. Am J Hypertens 2013; 26(3): 357-366.

7. Keller K, Stelzer K, Ostad MA, et al. Impact of exaggerated blood pressure response in normotensive individuals on future hypertension and prognosis: systematic review according to PRISMA guideline. Adv Med Sci 2017; 62(2): 317-329.

8. Smith RG, Rubin SA and Ellestad MH. Exercise hypertension: an adverse prognosis? J Am Soc Hypertens 2009; 3(6): 366-373.

9. Lauer MS, Pashkow FJ, Harvey SA, et al. Angiographic and prognostic implications of an exaggerated exercise systolic blood pressure response and rest systolic blood pressure in adults undergoing evaluation for suspected coronary artery disease. J Am Coll Cardiol 1995; 26(7): 1630-1636.

10. Hedberg P, Ohrvik J, Lonnberg I, et al. Augmented blood pressure response to exercise is associated with improved long-term survival in older people. Heart 2009; 95(13): 1072-1078.

11. Gupta MP, Polena S, Coplan N, et al. Prognostic significance of systolic blood pressure increases in men during exercise stress testing. Am J Cardiol 2007; 100(11): 1609-1613.

12. Campbell L, Marwick TH, Pashkow FJ, et al. Usefulness of an exaggerated systolic blood pressure response to exercise in predicting myocardial perfusion defects in known or suspected coronary artery disease. Am J Cardiol 1999; 84(11): 1304-1310.

13. Bevegård $\mathrm{S}$ and Danielson $\mathrm{M}$. Effect of body position on circulatory adaptation to exercise in patients with essential hypertension. Scand J Clin Lab Invest 1977; 37(1): 63-70.

14. Joyner MJ and Casey DP. Regulation of increased blood flow (hyperemia) to muscles during exercise: a hierarchy of competing physiological needs. Physiol Rev 2015; 95(2): 549-601.
15. Rowell LB, Brengelmann GL, Blackmon JR, et al. Disparities between aortic and peripheral pulse pressures induced by upright exercise and vasomotor changes in man. Circulation 1968; 37(6): 954-964.

16. Mandsager K, Harb S, Cremer P, et al. Association of cardiorespiratory fitness with long-term mortality among adults undergoing exercise treadmill testing. JAMA Netw Open 2018; 1(6): e183605.

17. Myers J, Prakash M, Froelicher V, et al. Exercise capacity and mortality among men referred for exercise testing. $N$ Engl J Med 2002; 346(11): 793-801.

18. Currie KD, Floras JS, La Gerche A, et al. Exercise blood pressure guidelines: time to re-evaluate what is normal and exaggerated? Sports Med 2018; 48(8): 1763-1771.

19. Boyle CA and Decoufle P. National sources of vital status information: extent of coverage and possible selectivity in reporting. Am J Epidemiol 1990; 131(1): 160-168.

20. Morris CK, Myers J, Froelicher VF, et al. Nomogram based on metabolic equivalents and age for assessing aerobic exercise capacity in men. J Am Coll Cardiol 1993; 22(1): 175-182.

21. Holmgren A. Circulatory changes during muscular work in man; with special reference to arterial and central venous pressures in the systemic circulation. Scand $J$ Clin Lab Invest 1956; 8(Suppl 24): 1-97.

22. Pressler A, Jahnig A, Halle M, et al. Blood pressure response to maximal dynamic exercise testing in an athletic population. J Hypertens 2018; 36(9): 1803-1809.

23. Williams B, Mancia G, Spiering W, et al. 2018 ESC/ESH guidelines for the management of arterial hypertension. Eur Heart J 2018; 39(33): 3021-3104.

24. Singh JP, Larson MG, Manolio TA, et al. Blood pressure response during treadmill testing as a risk factor for newonset hypertension. The Framingham heart study. Circulation 1999; 99(14): 1831-1836.

25. Jae SY, Franklin BA, Choo J, et al. Exaggerated exercise blood pressure response during treadmill testing as a predictor of future hypertension in men: a longitudinal study. Am J Hypertens 2015; 28(11): 1362-1367.

26. Berger A, Grossman E, Katz M, et al. Exercise blood pressure and the risk for future hypertension among normotensive middle-aged adults. $J$ Am Heart Assoc 2015; 4(4): e001710.

27. Schultz MG, Otahal P, Picone DS, et al. Clinical relevance of exaggerated exercise blood pressure. J Am Coll Cardiol 2015; 66(16): 1843-1845.

28. Weiss SA, Blumenthal RS, Sharrett AR, et al. Exercise blood pressure and future cardiovascular death in asymptomatic individuals. Circulation 2010; 121(19): 2109-2116.

29. Schultz MG, Otahal P, Cleland VJ, et al. A hypertensive response to exercise independently predicts cardiovascular events and mortality: a systematic review and metaanalysis. Artery Research 2012; 6: 160.

30. Allison TG, Cordeiro MA, Miller TD, et al. Prognostic significance of exercise-induced systemic hypertension in healthy subjects. Am J Cardiol 1999; 83(3): 371-375.

31. O'Neal WT, Qureshi WT, Blaha MJ, et al. Systolic blood pressure response during exercise stress testing: the Henry 
Ford Exercise Testing (FIT) Project. J Am Heart Assoc 2015; 4(5): e002050.

32. Filipovský J, Ducimetiere P and Safar ME. Prognostic significance of exercise blood pressure and heart rate in middle-aged men. Hypertension 1992; 20(3): 333-339.

33. Fagard R, Staessen J, Thijs L, et al. Prognostic significance of exercise versus resting blood pressure in hypertensive men. Hypertension 1991; 17(4): 574-578.
34. Mundal R, Kjeldsen SE, Sandvik L, et al. Exercise blood pressure predicts mortality from myocardial infarction. Hypertension 1996; 27(3 Pt 1): 324-329.

35. Kurl S, Laukkanen JA, Rauramaa R, et al. Systolic blood pressure response to exercise stress test and risk of stroke. Stroke 2001; 32(9): 2036-2041. 